\title{
Surface wave dispersion in cement- based media: Inclusion size effect
}

$\operatorname{AUTHOR}(S):$

AGGELIS, D.G.; SHIOTANI, T.

CITATION:

AGGELIS, D.G....[et al]. Surface wave dispersion in cement-based media: Inclusion size effect. NDT \& E International 2008, 41(5): 319-325

ISSUE DATE:

2008-07

URL:

http://hdl.handle.net/2433/93471

RIGHT:

Copyright @ 2008 Elsevier; この論文は出版社版でありません。引用の 際には出版社版をご確認ご利用ください。; This is not the published version. Please cite only the published version. 


\title{
Surface wave dispersion in cement-based media: inclusion size effect
}

\author{
D.G. Aggelis ${ }^{1}$ and T. Shiotani ${ }^{2 *}$ \\ ${ }^{1}$ Research Institute of Technology, Tobishima Corporation \\ 5472 Kimagase, Noda, Chiba, 270-0222, Japan \\ ${ }^{2}$ Department of Urban Management, Graduate School of Engineering, Kyoto University, C1-2-236, \\ Kyoto-Daigaku-Katsura, Nishikyo-Ku Kyoto 615-8540, Japan
}

\begin{abstract}
This paper presents the experimental study of surface wave propagation in cementitious material with different shape and size but same volume content of thin inclusions that simulate distributed damage. The Rayleigh wave velocity changes almost up to $20 \%$ depending on the inclusion shape, while the longitudinal velocity, the experimental variance, as well as the coherence of the signals is also affected. It is demonstrated that the material is strongly dispersive and caution should be taken for the interpretation of the wave measurements since the velocity is sensitive not only to the damage content but also to the "crack" size.
\end{abstract}

Keywords: Ultrasound, dispersion, concrete, scattering.

* Corresponding author. Tel:+81-75-383-3261, Fax:+81-75-383-3264. E-mail address: Shiotani@toshi.kuciv.kyoto-u.ac.jp 


\section{Introduction}

Wave propagation in heterogeneous media is a topic concentrating many efforts from engineers and scientists. Apart from the intellectual challenge, the main aim is quality characterization by means of correlations of propagation characteristics with material properties. In the area of concrete, although stress waves have been used for many decades [1], the results, concerning mainly distributed damage, cannot be considered satisfactory [2]. A certain reason is the nature of the material. Being a porous composite of different elastic and visco-elastic materials of different size distributions, renders the quantification of the influence of different propagation mechanisms troublesome. Recently, in order to enlighten wave propagation in these media, elaborate studies aimed to quantify the contribution of scattering and viscous mechanisms in concrete even at frequencies much higher than the applied at practical tests [3-10].

Generally, propagation of body waves in inhomogeneous media has been studied extensively, both theoretically and experimentally [11-15]. On the other hand, Rayleigh waves have been studied for layered media [16,17] or media with surface cracks [18-20]. However, to the authors' knowledge, the issue of Rayleigh wave propagation in a medium with randomly distributed and oriented thin inclusions has not been addressed neither experimentally, nor theoretically. In the present paper, the inhomogeneity size effect is studied through surface wave measurements in a cementitious matrix containing small plate inclusions of vinyl material in volume content of $1 \%$. The thin plate inclusions aim to simulate distributed damage more realistically than spherical ones that have been used previously. It is seen that considerable discrepancies in phase velocity curves arise with different inclusion size. Additionally, the coherence of the signals is also affected, as well as the experimental variance due to locality effects. Finally, Rayleigh waves seem to be more sensitive to 
the inclusions shape than longitudinal waves, as they undergo stronger velocity reduction and exhibit higher experimental variance.

For a case like the one discussed herein, a theoretical model is not available to provide a guide for the explanation of experimental behavior. Scattering, as well as viscous mechanisms are certain to influence but their contribution cannot be exactly quantified. However, original and valuable conclusions can be drawn highlighting the dispersive nature of cementitious material that so far is poorly discussed or even ignored.

\section{Experiment}

\subsection{Materials}

The shape of the cementitious specimens was cubic with $150 \mathrm{~mm}$ side. The water to cement ratio of mortar was 0.5 by mass, the sand to cement ratio 2 by mass and the maximum size of sand was 3 $\mathrm{mm}$. The mortar ingredients (cement powder, sand and water) were mixed in a concrete mixer for two minutes. Afterwards, the vinyl inclusions (accounting to $1 \%$ of each specimen's volume) were added and the mixer worked for two additional minutes, before the material was cast in metal forms. After twenty four hours the specimens were demolded and cured in tap water for 28 days. The tests were conducted on dry specimens.

The ratio of acoustic impedances of the two constituents (mortar and vinyl) is approximately 8 , high enough to expect strong scattering phenomena. Four different shapes of vinyl inclusions were used, namely $15 \times 15 \times 0.5 \mathrm{~mm}, 15 \times 15 \times 0.2 \mathrm{~mm}, 30 \times 30 \times 0.5 \mathrm{~mm}$ and $30 \times 30 \times 0.2 \mathrm{~mm}$, one for each specimen. The thickness of $0.2 \mathrm{~mm}$ and $0.5 \mathrm{~mm}$ are typical for concrete cracks. Hereafter, when referring to the 
inclusion shape, the unit (mm) will be omitted for brevity. Changing the size or the thickness affects the total number of the inclusions inside each specimen since the volume content is constant. Specifically, considering the size of 30x30x0.5, 75 individual vinyl inclusions account for $1 \%$ of the specimen's volume. The number is increased for $30 \times 30 \times 0.2$ and $15 \times 15 \times 0.5$ by factors of 2.5 and 4 respectively. Finally, concerning the smallest size $15 \times 15 \times 0.2$, ten times more inclusions were included in the specimen, specifically 750. The characteristics of the inclusions are summarized in Table 1, along with other parameters that will be used later.

The dispersion of the fillers in the matrix is considered random. The important point in such a case is that no conglomeration occurs, that would severely influence the local concentration at different parts of the specimens. After examination of a pilot specimen (see Fig. 1) no conglomeration was observed. The actual spatial and orientation distribution of the inclusions is not confirmed or measured. It is noted however, that the distribution of cracks in the actual material is also unknown. The cracks do not have a uniform pattern but are distributed in a more random way. For the materials at hand (and in case the distribution was uniform and the scatterers spherical) the space between individual inclusions can be calculated and is included in the last column of Table 1, as a rule of thumb. It is seen that although the total volume content of vinyl is the same, the different size governs the total number of inclusions, the distance between them and finally how many times the wave will encounter scatterers once it has been introduced by the pencil lead break until it is captured by the last receiver.

\subsection{Ultrasonic measurements}

Three broadband piezoelectric transducers, Fujiceramics 1045S, were placed on the specimens' surface, in a straight line, with center to center distance of $20 \mathrm{~mm}$. The excitation was introduced by 
pencil lead break that produces a bandwidth of $0-200 \mathrm{kHz}$, at a distance $20 \mathrm{~mm}$ in front of the first receiver. For this band, more sensitive, resonant sensors could be used; however, broad band transducers were the most reliable choice since the aim was not a simple pulse velocity measurement but also more elaborate dispersion characterization. The signals were digitized with a sampling rate of $10 \mathrm{MHz}$ and acquired in a Mistras system of Physical Acoustics Corp. The surfaces of all specimens had the same good finishing since the same metal molds were used and silicone grease was applied between the sensor and the specimen surface to enhance acoustical coupling. The separation distances between excitation and sensors were kept constant but their location was changed in order to check locality effects. After each measurement the "line" of excitation and receiver points was slightly translated and/or rotated in the center area of the surface and not closer than $30 \mathrm{~mm}$ from the edge (the total area of the surface is $150 \times 150 \mathrm{~mm}$ ). In total, more than 20 measurements were conducted on each specimen. Measurements were not expanded to larger area in order to avoid any possibility of edge effects. Although more measurements could be conducted on the center area, they would certainly include increased overlap. In any case, the twenty measurements were sufficient to reveal the difference in experimental variance that characterizes the different specimens as will be seen.

\section{Results and discussion}

\subsection{Waveform distortion}

Heterogeneity induces velocity and attenuation variation with pulse frequency, inclusion content and shape as well as distortion of the received pulse in time domain [21]. Before discussing velocity, it is interesting to focus on the waveform distortion. In a homogeneous non dispersive half-space, a pulse propagating on the surface will undergo little or no distortion, since the nature of the waves is non 
dispersive. On the contrary, in an inhomogeneous medium, scattering will distribute the energy at various paths with different lengths, causing visible distortion in each waveform. However, since a part of energy can survive being always scattered in the forward direction, a common pattern is expected to exist in all the individual waveforms [21,22]. This is known as the coherent or ballistic pulse and can be extracted by averaging of several individual waveforms. On the other hand, due to the random character of scattering, the rest scattered components tend to be eliminated by averaging.

In Fig. 2(a) the average waveforms collected by each sensor on a plain mortar specimen surface are depicted. It is seen that after the initial longitudinal arrivals, a strong Rayleigh cycle is clearly observed in all three distances. In Figs. 2(b), (c) and (d) the corresponding waveforms from media with $1 \%$ inclusions can be seen. For the cases of $15 \times 15 \times 0.5$ and $30 \times 30 \times 0.5$, see Fig. 2(b) and (c) respectively, the Rayleigh cycle can still be followed throughout the propagation although it exhibits longer duration and its shape is not a sharp as in plain mortar. For the case of 30x30x0.2 depicted in Fig. 2(d) however, the Rayleigh cycle is extremely distorted even from the second transducer, while at the distance of $60 \mathrm{~mm}$ from the excitation, see bottom waveform of Fig. 2(d), the coherent field is so weak that the Rayleigh cycle can hardly be identified. This can be attributed to the size as well as the number of inclusions present at the wavepath between the excitation and the receivers. Indeed, although the total volume fraction of the inclusions was always the same, changing the size of the inclusions results in different total number of inclusions (a few thick as opposed to many thin ones), as mentioned earlier. Therefore, for the case of Fig. 2(d) of 30x30x0.2 mm, a much larger number of inclusions correspond to the $1 \%$ volume fraction than the case of $30 \times 30 \times 0.5 \mathrm{~mm}$ thickness depicted in Fig. 2(c). This means more encounters of the wavefront with scatterers and seems reasonable to result in stronger waveform distortion. It is also mentioned that apart from the shape, size and number of inclusions, their orientation is another factor influencing the propagation. In case the 
propagating wave faces the plate side of the inclusion at normal angle, it is reasonable to expect strong reflection due to the impedance mis-match, while in case the thin inclusion is positioned parallel to the propagation direction the influence should be much different. Considering also the range of possible angles between the propagation direction and the inclusions, it is reasonable to expect increased randomness of the phenomenon, compared to the spherical scatterer case [6] for which, each one of them has the same influence.

In relevant studies with spherical shape of inclusions, it is straightforward to refer to the dimensionless wavelength (wavelength divided by the scatterer diameter). In this case however, such a descriptor is not readily available due to the plate shape of the inclusions, with equal height and width but much smaller thickness. This is much closer to the actual crack shape and thus makes the study realistic; however renders any normalization difficult. Apart from that, as seen in Fig. 2, the Rayleigh wave cycle broadens with propagation. This has an effect on the frequency and therefore, also on the propagating wavelength. According to the frequencies exhibited in this study, the dominant wavelength could be less than $20 \mathrm{~mm}$ for plain mortar but more than $40 \mathrm{~mm}$ for mortar with inclusions after $60 \mathrm{~mm}$ of propagation. Therefore, it would not be safe to refer to one specific wavelength; however, in any case two of the three dimensions of the inclusions are of the same order with the wavelength while the thickness is two orders of magnitude lower.

As seen, the coherent part of the waveform is certainly influenced by the presence of inclusions as well as, more importantly, by their shape. This can be examined by the "coherence" function that underlines the similarities of two signals in their frequency content [23-25] and is given by

$\gamma_{x y}^{2} \boldsymbol{\Phi}=\frac{\left|G_{x y} \boldsymbol{f}\right|^{2}}{G_{x x} \boldsymbol{f} G_{y y} \boldsymbol{f}_{-}^{-}} \quad 0 \leq \gamma_{x y}^{2} \boldsymbol{f}_{-}^{-}$ 
where $G_{x y}(f)$ is the cross-spectral density function between two waveforms $X(t)$ and $Y(t)$ and $G_{x x}(f)$, $\mathrm{G}_{\mathrm{yy}}(\mathrm{f})$ are the autospectral density functions of $\mathrm{X}(\mathrm{t})$ and $\mathrm{Y}(\mathrm{t})$, respectively.

Specifically, the coherence function was calculated after each excitation between signals of the first and the third transducer, which are $40 \mathrm{~mm}$ apart. The results for all the materials are presented in Fig. 3. Again, each curve comes from the average of 20 individual coherence curves calculated for different pairs of waveforms. It is seen that the coherence of signals in plain mortar is the highest, with an average value throughout the first $200 \mathrm{kHz}$ almost 0.99 . The coherence for the specimens with thick inclusions $(0.5 \mathrm{~mm})$ is quite high (especially for the 30x30x0.5), averaging to approximately 0.97 . This is reasonable since the number of these large inclusions is very low (75 inclusions in the whole specimen) and the average distance between them is calculated at $35 \mathrm{~mm}$, as stated in Table 1. Therefore, the number of scatterers between the excitation and the receiver is expected to be limited compared to other thin inclusions which are contained in larger numbers. Accordingly, the inclusions of $0.2 \mathrm{~mm}$ thickness exhibit much lower coherence with average value approximately at 0.85 . This shows again that the propagation is sensitive to different shape, size and number of inclusions, while the multiple scattering drastically weakens the coherent part of the signal as it propagates through the inhomogeneous medium, even for the low frequencies below 200 $\mathrm{kHz}$ which are commonly used for concrete testing.

\subsection{Pulse velocity}

Surface wave propagation is complicated since different kinds of waves are present. Normally the Rayleigh waves occupy most of the energy, but the longitudinal are the fastest type. Therefore, measuring the transit time of the first detectable disturbance of the waveform, leads to the calculation of the longitudinal wave velocity. In concrete testing, this is referred to as pulse velocity 
$[9,10]$ and although it cannot be considered either phase or group velocity, it is widely used for rough correlations with quality. It is mentioned that in literature, phase velocity is calculated either by some characteristic point in the middle of a tone-burst of a specific frequency $[3,12]$, or by spectral analysis of a broadband pulse [9,26,27] On the other hand, group velocity is calculated by the maximum peak [28], the maximum of the wave envelope [21], or cross-correlation between the "input" and "output" waveforms [29]. In homogeneous media all these measurements are expected to lead to the same value. However, for concrete it has been shown that these velocities are not necessarily close $[9,29]$.

In Fig. 4(a) the longitudinal wave velocity is depicted for different types of inclusions. The velocity values are normalized to the velocity of plain mortar that was measured at $3976 \mathrm{~m} / \mathrm{s}$. As observed, the different size has considerable effect on the velocity since the thick inclusions reduce the velocity to approximately $97.5 \%$ of plain mortar and thin inclusions reduce the velocity even to $93 \%$. This shows that the material cannot be treated as homogeneous considering only the volume fraction of inclusions (or cracks in an actual situation), since their shape and size is also important. The velocities presented come from the average of 20 individual measurements. Variation effects will be discussed later.

As to the Rayleigh wave propagation, if a reference point can be identified, e.g. the strong negative peaks in Fig. 2(a), the Rayleigh velocity can be calculated easily. However, in case of strong dispersion, case of Fig. 2(d) a reference point cannot be used. Therefore, an approach with crosscorrelation was followed. The time lag resulting after cross correlation between the signals of the first and third transducer (40 $\mathrm{mm}$ separation), gives a measure of the velocity with which the energy 
propagates and could be considered close to the group velocity [29]. For this task the first $100 \mu$ s of the waveforms were used where the Rayleigh contribution is certain to exist.

The results are shown again in Fig. 4(a). The plain mortar velocity was $2141 \mathrm{~m} / \mathrm{s}$. The Rayleigh velocity follows similar diminishing trend with the longitudinal, being however more strongly reduced, to even $82 \%$ of the plain mortar, for the case of $30 \times 30 \times 0.2$ inclusions. This again shows the importance of the scatterer type. Additionally, the differential influence of inclusion shape on longitudinal and Rayleigh wave velocities should be highlighted. This is another indication of the inhomogeneous nature of the material. The addition of lower elasticity inclusions reduces the "effective" modulus of elasticity of mortar. In case the material could be regarded as homogeneous, the influence on longitudinal and Rayleigh velocities should be the same, since they are both firmly connected to the elastic properties. However, for the material at hand, the Rayleigh is obstructed much more intensively, especially by the thin inclusions, showing that a traditional homogenized approach used for concrete is not adequate even for $1 \%$ of inclusions. This should also be related to the propagation mechanism of Rayleigh waves, which includes displacement components in two directions; parallel, as well as normal to the direction of propagation, being therefore more sensitive to inhomogeneity.

As stated earlier, the velocity values come from the average of 20 individual measurements. The experimental variance of these measurements is directly connected with the inclusion type. In Fig. 4(b) the coefficient of variation, or the standard deviation divided by the average value, is presented for the velocity values of Fig. 4(a). As expected, the plain mortar exhibits the lowest variation both for longitudinal $(2.56 \%)$ and Rayleigh waves $(3.03 \%)$. This is the result of the limited inhomogeneity of the material, relatively to the wavelength. Indeed, the maximum sand grain size 
used in mortar was $3 \mathrm{~mm}$, approximately one order of magnitude shorter than the wavelength. However, the presence of vinyl inclusions increases drastically the experimental variance. For the case of $30 \times 30 \times 0.2$ inclusions, the coefficient of variation is $6.61 \%$ for longitudinal and more than $20 \%$ for Rayleigh. This shows not only the inhomogeneity influence but also the locality of such a medium. Although the inclusion content was strictly controlled in total, it is reasonable that the local arrangement of inclusions is different both in content and orientation, strongly influencing the acquired waveforms from point to point. From Figs. 4(a) and (b) it is evident that the reason that the inclusion size affects velocity, affects the coefficient of variation as well. In any case, care should be taken to ensure that measurements are representative, especially in "damaged" material.

\subsection{Phase velocity}

The above mentioned approach yields a measure of the velocity of the whole pulse. In a heterogeneous medium like the one studied herein, the velocity is expected to be strongly dependent on the frequency. Therefore, it is of certain value to calculate the dispersion curve. In the case of Rayleigh waves this is not trivial since there are always contributions from longitudinal and shear waves that are faster. Therefore, the Rayleigh cannot be isolated. However, since it generally carries more energy than the other types, concentrating on a time window where the Rayleigh is expected, can yield information about this wave with little influence from other types. In this case a window of $30 \mu$ s located around the major Rayleigh arrivals was isolated and the rest of the waveform was zeropadded as presented in similar cases [26]. Using Fast Fourier Transform, the phase of the waveform is calculated and unwrapped. Therefore, the difference of phase between waveforms collected at different distances from the excitation (i.e. the first and third receiver) leads to the calculation of phase velocity vs. frequency curve [27]. The results are depicted in Fig. 5 for materials with different 
inclusion type. Each curve is the average of 20 individual curves in order to diminish variation effects.

It is seen that even plain mortar exhibits dispersive behavior with velocity increasing throughout the first $200 \mathrm{kHz}$. This has been mentioned for longitudinal waves of sound mortar and concrete previously [9]. It is interesting to see that the dispersion curve for inclusions with dimensions $15 \times 15 \times 0.5$ almost does not affect the curve, being slightly lower in average value. However, other inclusion shapes seem to have stronger influence, with the large and thin inclusions $(30 \times 30 \times 0.2)$ lowering the curve by more than $700 \mathrm{~m} / \mathrm{s}$ in average. Additionally, all the curves and especially three of them exhibit strong velocity increase for the band up to $50 \mathrm{kHz}$ or $60 \mathrm{kHz}$. For higher frequencies each curve seems to converge. This is a behavior generally observed in composite systems. For low frequencies, the velocity may exhibit strong variations or resonance peaks. However, as the frequency increases, the variations seem to diminish $[11,12,30,31]$ as is the case for this study. In general, a dispersion peak is observed when the wavelength is approximately 3 to 4 times the size of the scatterer, in case of spherical shape. As stated above, in the present case this kind of relation is difficult to acquire. However, the wavelength at the frequency of the dispersion peak can be calculated at approximately $35 \mathrm{~mm}$, which is approximately 4-6 times the diameter of the equivalent spheres, as seen in Table 1.

It is mentioned that the level of the dispersion curve is not necessarily close to the value of velocity measured by cross-correlation. For plain mortar, the Rayleigh phase velocity curve seen in Fig. 5, averages at $2073 \mathrm{~m} / \mathrm{s}$ being close to the "group" velocity of $2141 \mathrm{~m} / \mathrm{s}$. However, as the inhomogeneity increases, considerable discrepancies arise. Indicatively, for the inclusions of 30x30x0.2 the phase velocity curve averages at $1331 \mathrm{~m} / \mathrm{s}$, as seen in Fig. 5, while the cross- 
correlation leads to a velocity of $1765 \mathrm{~m} / \mathrm{s}$, see Fig. 4(a). This discrepancy has been observed in other cases of cementitious materials and increases with the level of inhomogeneity, i.e. from cement paste to mortar with sand grains or concrete with large aggregates [9], as well as other strongly scattering material [21]. This trend is reasonable since only in homogeneous, non-dispersive materials group and phase velocities are equal.

Finally, a certain concern in this kind of measurements is the coupling of the piezoelectric transducers to the specimen surface. Although the coupling conditions cannot be absolutely identical in each measurement, it seems that any effect in this laboratory series was insignificant compared to the material's response itself. It is indicative that the coefficient of variation was less than $3 \%$ for the velocity of plain mortar. This variation can be attributed to both experimental errors and weak locality effects of mortar. However, the experimental variance strongly increased for other types of material although the measurement procedure was the same. This certainly demonstrates that any measurement error does not mask the material's response.

\section{Conclusion}

This paper presents experimental data in the field of surface wave propagation through a strongly inhomogeneous medium with randomly distributed and oriented thin inclusions simulating cracks. Specifically, the study focuses on the influence of inclusion size. Thin plastic inclusions were embedded in a cementitious matrix to simulate distributed damage. The results demonstrate that the propagation behavior of cementitious material with simulated damage is far from homogeneous. For the same low inclusion volume fraction of $1 \%$, considerable discrepancies arise according to the size of the inclusions. These discrepancies concern the shape of time domain waveforms, the group and 
phase velocities of Rayleigh, as well as the longitudinal waves. Rayleigh waves are more sensitive to the inclusions than longitudinal since they undergo greater decrease and exhibit higher experimental variance, due to the locality of the medium.

It seems that scattering is a crucial mechanism of propagation, since not only the volume fraction, but also the shape of the inclusions strongly influences wave propagation. However, both the constituent materials in this study have visco-elastic character. Therefore, the exact contribution of the different mechanisms can be characterized accurately only with the support of a theoretical model.

The present study demonstrates the dispersive behavior of cementitious material that so far has been underestimated. It is seen that the influence of inhomogeneity shape and size, is apparently one of the reasons that make the establishment of a single relation between pulse velocity and damage of concrete troublesome. Nevertheless, exploitation of the characteristics of scattered waves can provide valuable information since inclusion or crack parameters like shape, size and content influence the time domain waveforms and the dispersion curves. 


\section{References}

[1] Kaplan MF. The effects of age and water/cement ratio upon the relation between ultrasonic pulse velocity and compressive strength. Mag. Con. Res. 1959;11(32):85-92.

[2] Popovics S. Analysis of the Concrete Strength versus Ultrasonic Pulse Velocity Relationship. Materials Evaluation 2001;59(2):123-130.

[3] Aggelis DG, Polyzos D, Philippidis TP. Wave dispersion and attenuation in fresh mortar: theoretical predictions vs. experimental results. Journal of the Mechanics and Physics of Solids 2005;53(4):857-883.

[4] Jacobs LJ, Owino JO. Effect of aggregate size on attenuation of Rayleigh surface waves in cement-based materials. J. Eng. Mech.-ASCE 2000;126(11):1124-1130.

[5] Becker J, Jacobs LJ, Qu J. Characterization of cement-based material using diffuse ultrasound. J. Eng. Mech.-ASCE 2003;129(12):1478-1484.

[6] Chaix JF, Garnier V, Corneloup G. Ultrasonic wave propagation in heterogeneous solid media: Theoretical analysis and experimental validation. Ultrasonics 2006;44:200-210.

[7] Punurai W, Jarzynski J, Qu J, Kurtis KE, Jacobs LJ. Characterization of entrained air voids in cement paste with scattered ultrasound. NDT\&E International 2006;39(6):514-524.

[8] Punurai W, Jarzynski J, Qu J, Kurtis KE, Jacobs LJ. Characterization of dissipation losses in cement paste with diffuse ultrasound. Mechanics Research Communications 2007;34(3):289294.

[9] Philippidis TP, Aggelis DG. Experimental study of wave dispersion and attenuation in concrete. Ultrasonics 2005;43(7):584-595.

[10] Aggelis DG, Philippidis TP. Ultrasonic wave dispersion and attenuation in fresh mortar. NDT \& E International, 2004;37(8):617-631.

[11] Aggelis DG, Tsinopoulos SV, Polyzos D. An Iterative Effective Medium Approximation 
(IEMA) for wave dispersion and attenuation predictions in particulate composites, suspensions and emulsions. J. Acoust. Soc. Am. 2004;116(6):3443-3452.

[12] Kinra VK, Rousseau CQ. Acoustical and optical branches of wave propagation. Some additional results. J. Wave Mater. Interaction 1987;2:141-152.

[13] Ying CF, Truell R. Scattering of a plane longitudinal wave by a spherical obstacle in an isotropically elastic solid. J. Appl. Phys. 1956;27:1086-1097.

[14] Yang L, Turner A. Scattering of elastic waves in damaged media. J. Acoust. Soc. Am. 2003;113:2992-3000.

[15] Yang L, Turner A. Wave attenuations in solids with perfectly aligned cracks. Acoustics Research Letters Online 2005;6:99-105.

[16] Sansalone M, Carino NJ. Stress wave propagation methods. In: Malhotra VM, Carino NJ (Eds.). CRC Handbook on Nondestructive Testing of Concrete, Florida, CRC Press, 1991. p. 275-304.

[17] Kim DS, Seo WS, Lee KM. IE-SASW method for nondestructive evaluation of concrete structure, NDT\&E International 2006;39:143-154.

[18] Van Wijk K, Komatitsch D, Scales JA, Tromp J. Analysis of strong scattering at the microscale. J. Acoust. Soc. Am. 2004;115:1006-1011.

[19] Hevin G, Abraham O, Pedersen HA, Campillo M. Characterisation of surface cracks with Rayleigh waves: a numerical model. NDT\&E International 1998;31(4):289-297.

[20] Pecorari C. Rayleigh wave dispersion due to a distribution of semi-elliptical surface-breaking cracks. J. Acoust. Soc. Am. 1998;103:1383-1387.

[21] Cowan ML, Beaty K, Page JH, Zhengyou L, Sheng P. Group velocity of acoustic waves in strongly scattering media: Dependence on the volume fraction of scatterers. Phys. Rev. E 1998;58:6626-6636. 
[22] Page JH, Schriemer HP, Jones IP, Sheng P, Weitz DA. Classical wave propagation in strongly scattering media. Physica A 1997;241:64-71.

[23] Bendat JS, Piersol AG, Engineering Applications of Correlation and Spectral Analysis, 2nd ed., New York, Wiley, 1993.

[24] Grosse C, Reinhardt H, Dahm T. Localization and classification of fracture types in concrete with quantitative acoustic emission measurement techniques. NDT\&E International $1997 ; 30(4): 223-230$.

[25] Philippidis TP, Aggelis DG. An acousto-ultrasonic approach for the determination of waterto-cement ratio in concrete. Cement and Concrete Research 2003;33:525-538.

[26] Dokun OD, Jacobs LJ, Haj-Ali RM. Ultrasonic monitoring of material degradation in FRP composites. J. Eng. Mech.-ASCE 2000;126:704-710.

[27] Sachse W, Pao YH. On the determination of phase and group velocities of dispersive waves in solids, J. Appl. Phys. 1978;49:4320-4327.

[28] Washer GA, Green RE, Pond RB Jr. Velocity constants for ultrasonic stress measurement in prestressing tendons. Res. Nondestr. Eval. 2002 ;14:81-94.

[29] Aggelis DG, Shiotani T. Experimental study of surface wave propagation in strongly heterogeneous media. J. Acoust. Soc. Am. 2007;122(5):EL151-157.

[30] Kim JY, Ih JG, Lee BH. Dispersion of elastic waves in random particulate composites. J. Acoust. Soc. Am. 1995;97:1380-1388.

[31] Temkin S. Attenuation and dispersion of sound in dilute suspensions of spherical particles. J. Acoust. Soc. Am. 2000;108:126-146. 
Table 1. Characteristics of the four types of inclusions

\begin{tabular}{|c|c|c|c|c|c|c|c|}
\hline $\begin{array}{c}\text { Length } \\
(\mathrm{mm})\end{array}$ & $\begin{array}{c}\text { Width } \\
(\mathrm{mm})\end{array}$ & $\begin{array}{c}\text { Thickness } \\
(\mathrm{mm})\end{array}$ & $\begin{array}{c}\text { Vol. } \\
\text { content } \\
(\%)\end{array}$ & $\begin{array}{c}\text { Total number } \\
\text { of inclusions } \\
\text { in specimen }\end{array}$ & $\begin{array}{c}\text { Volume of } \\
\text { one } \\
\text { inclusion } \\
\left(\mathrm{mm}^{3}\right)\end{array}$ & $\begin{array}{c}\text { Diameter of } \\
\text { equivalent } \\
\text { sphere }(\mathrm{mm})\end{array}$ & $\begin{array}{c}\text { Distance } \\
\text { between } \\
\text { neighbor } \\
\text { particles } \\
(\mathrm{mm})^{*}\end{array}$ \\
\hline 15 & 15 & 0.2 & 1 & 750 & 45 & 4.4 & 17 \\
\hline 15 & 15 & 0.5 & 1 & 300 & 112.5 & 6.0 & 22 \\
\hline 30 & 30 & 0.2 & 1 & 188 & 180 & 7.0 & 26 \\
\hline 30 & 30 & 0.5 & 1 & 75 & 450 & 9.6 & 36 \\
\hline
\end{tabular}

*Center to center distance, assuming uniform distribution of volume-equivalent spheres. 


\section{Figure Captions}

Fig. 1 Dispersion of inclusions in a pilot specimen with 5\% vinyl.

Fig. 2 Averaged waveforms collected at $20 \mathrm{~mm}$ intervals on the surface of plain mortar (a), and mortar with $1 \%$ inclusions of shape: $15 \times 15 \times 0.5$ (b), 30x30x0.5 (c), 30x30x0.2 (d). (The shape is expressed in $\mathrm{mm}$ )

Fig. 3 Coherence function of waveforms in mortar with $1 \%$ inclusions of various shapes. (The shape is expressed in $\mathrm{mm}$ )

Fig. 4 (a) Normalized velocity of mortar with $1 \%$ inclusions of various shapes, (b) coefficient of variation for the values of (a). (The shape is expressed in $\mathrm{mm}$ ).

Fig. 5 Phase velocity vs. frequency for mortar with $1 \%$ inclusions of various shapes. (The shape is expressed in $\mathrm{mm})$. 


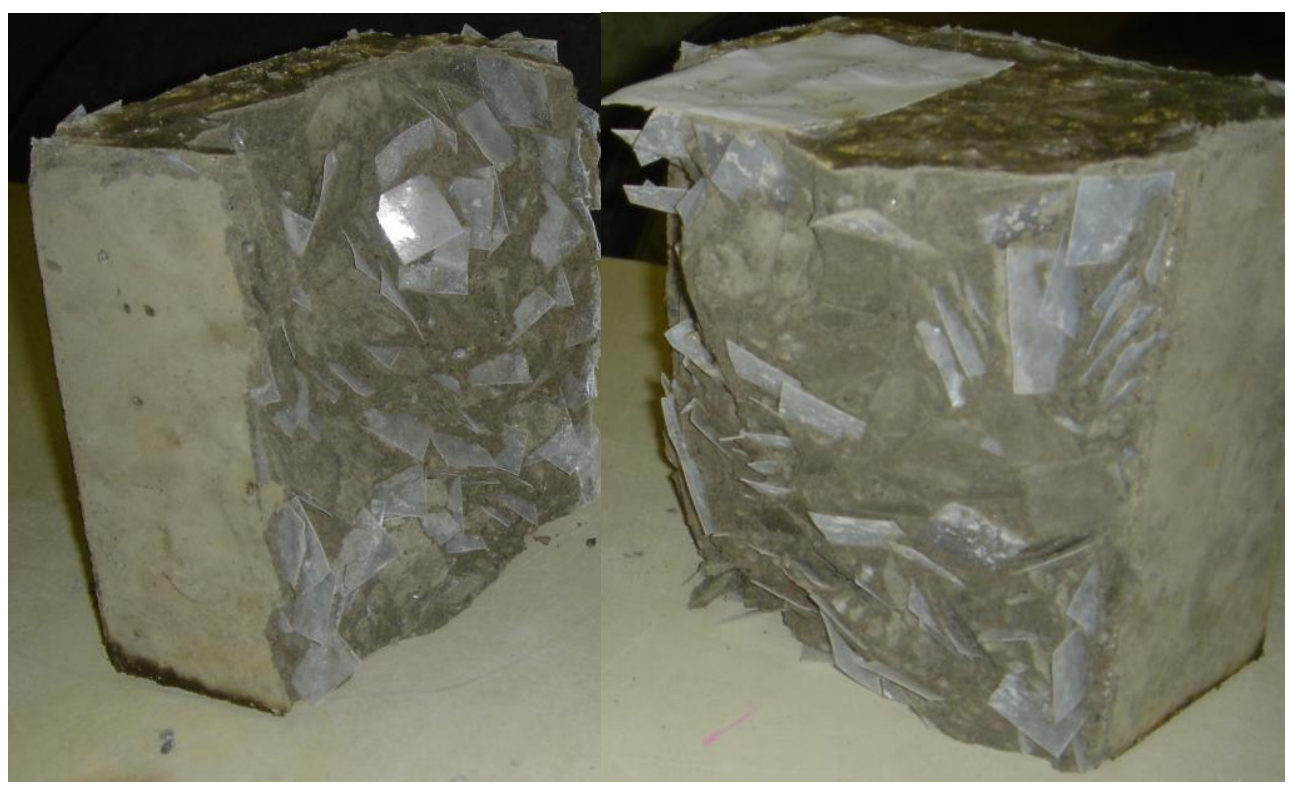

Fig. 1. Dispersion of inclusions in a pilot specimen with $5 \%$ vinyl. 


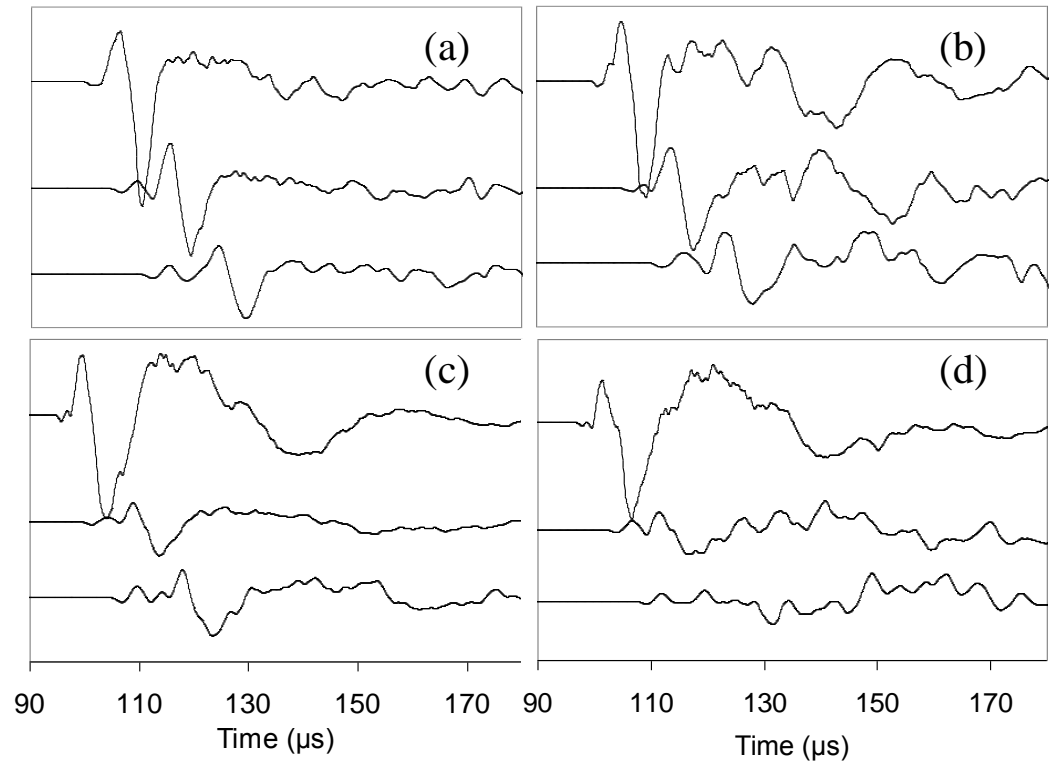

Fig. 2 Averaged waveforms collected at $20 \mathrm{~mm}$ intervals on the surface of plain mortar (a), and mortar with $1 \%$ inclusions of shape: $15 \times 15 \times 0.5$ (b), 30x30x0.5 (c), 30x30x0.2 (d). (The shape is expressed in $\mathrm{mm}$ ) 


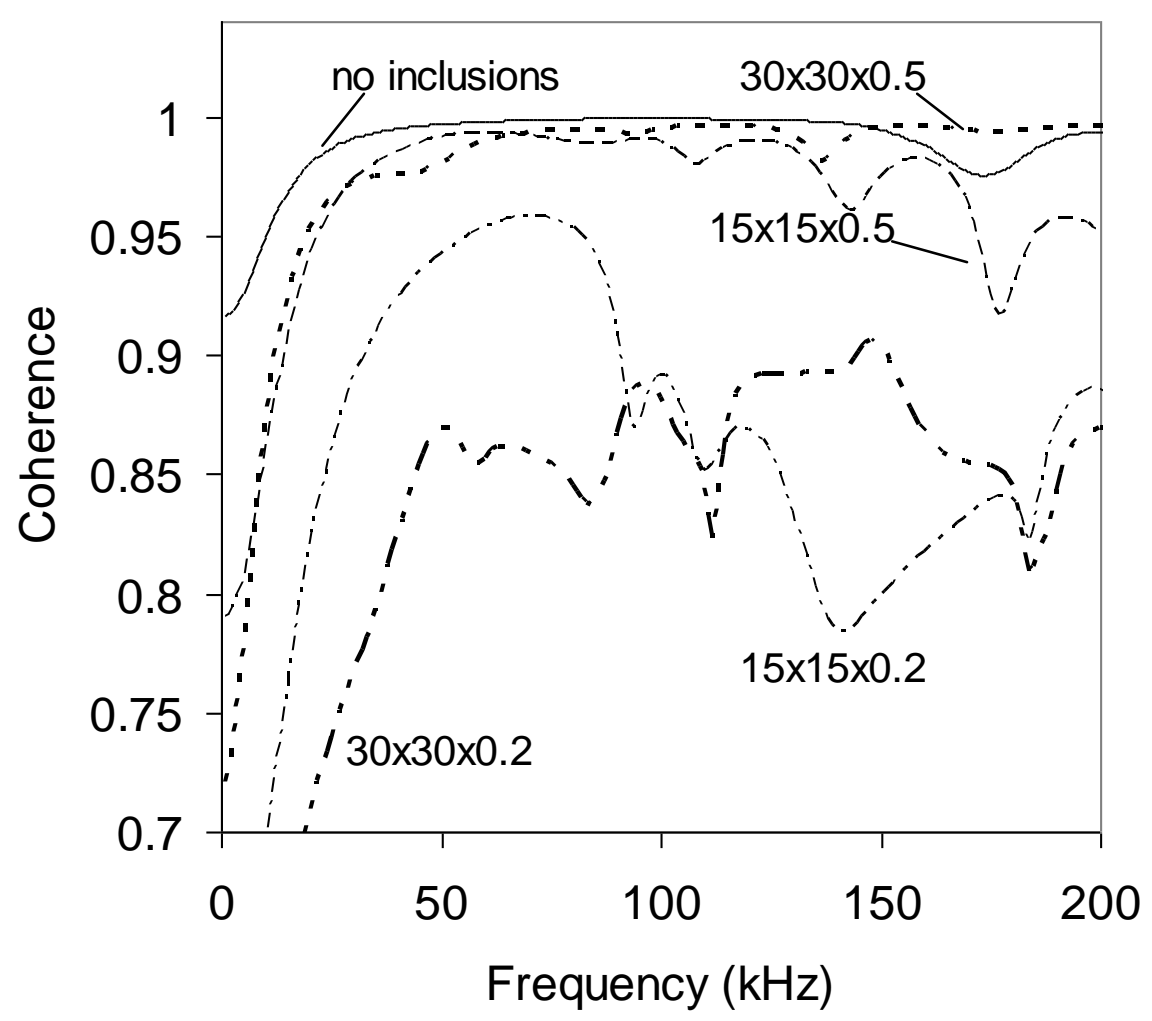

Fig. 3 Coherence function of waveforms in mortar with $1 \%$ inclusions of various shapes. (The shape is expressed in $\mathrm{mm}$ ) 

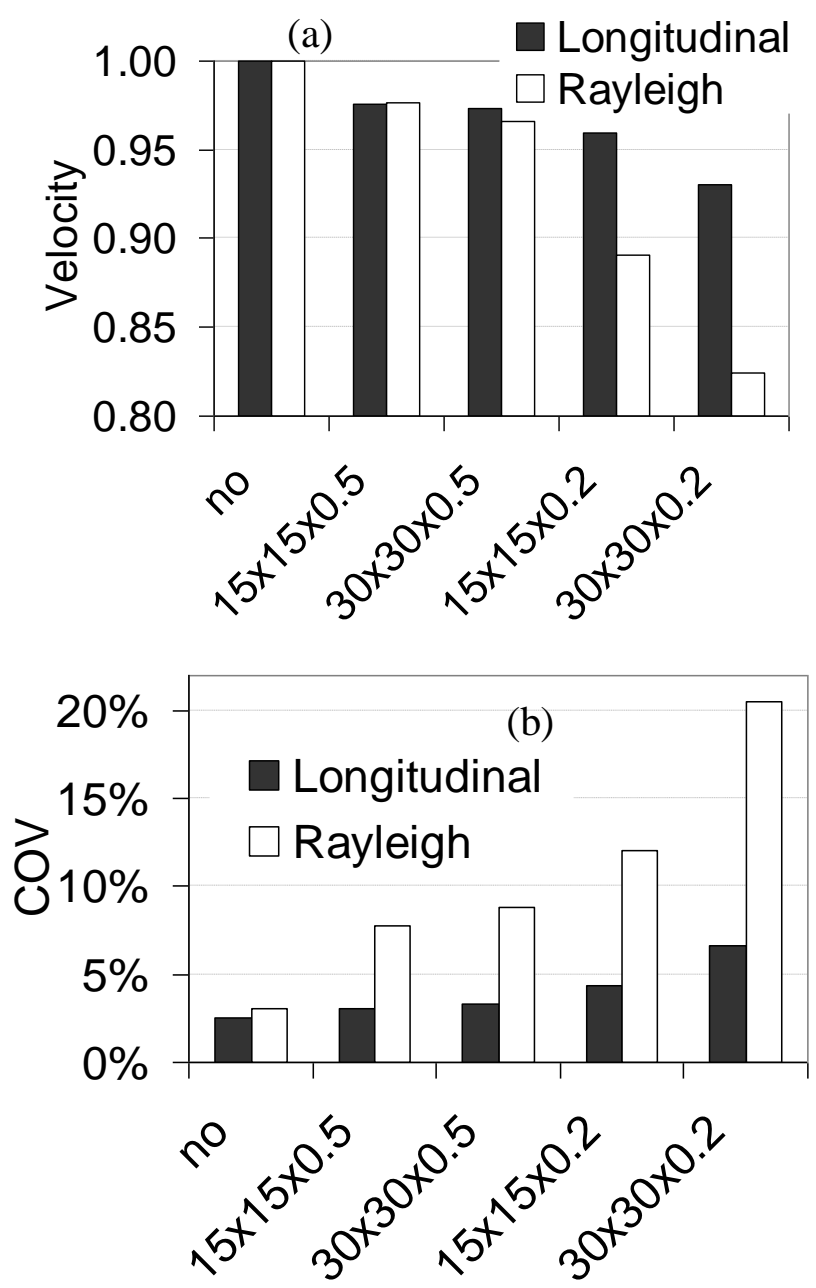

Fig. 4 (a) Normalized velocity of mortar with $1 \%$ inclusions of various shapes, (b) coefficient of variation for the values of (a). (The shape is expressed in $\mathrm{mm}$ ). 


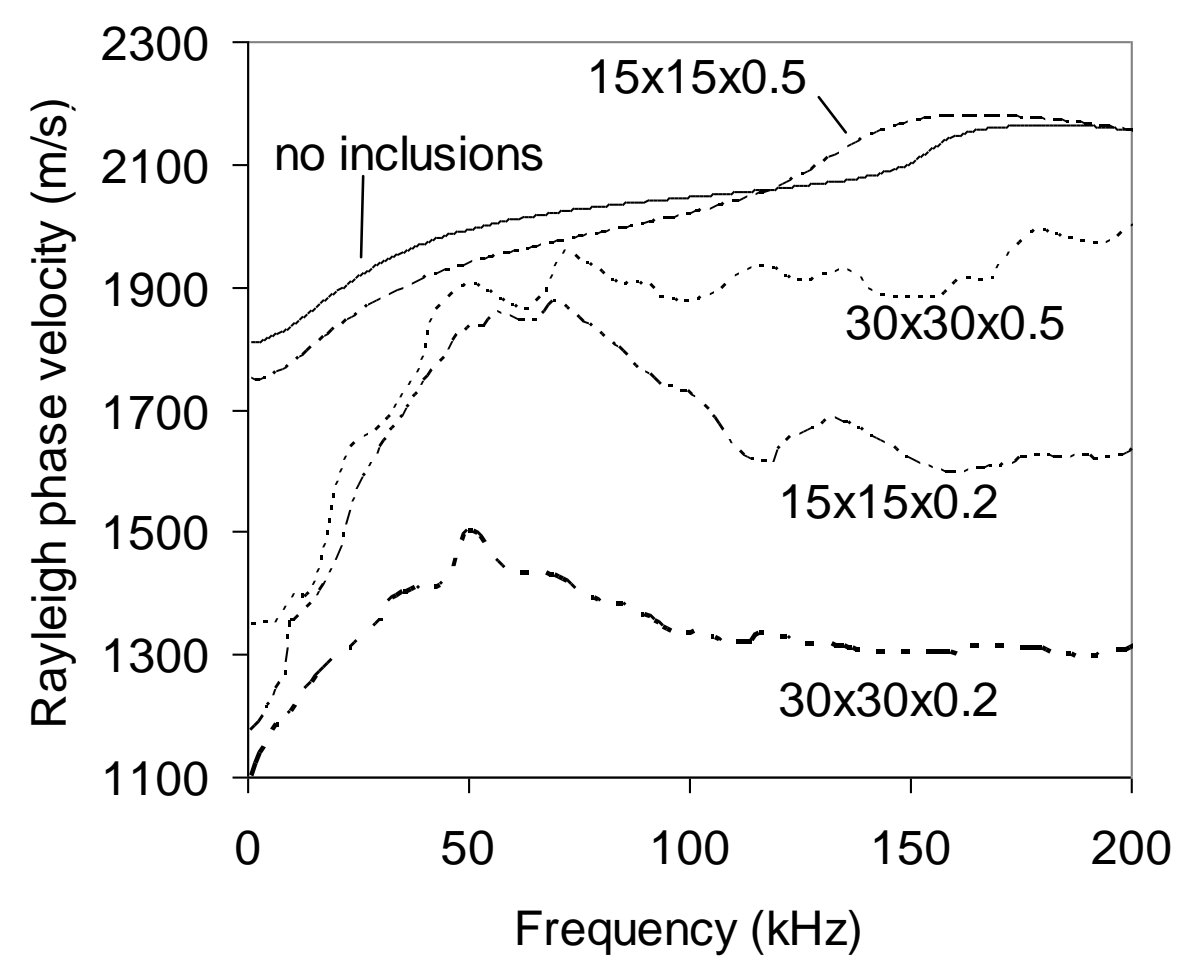

Fig. 5 Phase velocity vs. frequency for mortar with $1 \%$ inclusions of various shapes. (The shape is expressed in $\mathrm{mm}$ ) 